\title{
Moderate intensity supine exercise causes decreased cardiac volumes and increased outer volume variations: a cardiovascular magnetic resonance study
}

\author{
Katarina Steding-Ehrenborg ${ }^{1,2}$, Robert Jablonowski ${ }^{3}$, Per M Arvidsson ${ }^{3}$, Marcus Carlsson $^{3}$, Bengt Saltin ${ }^{1}$ \\ and Håkan Arheden ${ }^{3 *}$
}

\begin{abstract}
Background: The effects on left and right ventricular (LV, RV) volumes during physical exercise remains controversial. Furthermore, no previous study has investigated the effects of exercise on longitudinal contribution to stroke volume (SV) and the outer volume variation of the heart. The aim of this study was to determine if LV, RV and total heart volumes (THV) as well as cardiac pumping mechanisms change during physical exercise compared to rest using cardiovascular magnetic resonance (CMR).
\end{abstract}

Methods: 26 healthy volunteers ( 6 women) underwent CMR at rest and exercise. Exercise was performed using a custom built ergometer for one-legged exercise in the supine position during breath hold imaging. Cardiac volumes and atrio-ventricular plane displacement were determined. Heart rate (HR) was obtained from ECG.

Results: HR increased during exercise from $60 \pm 2$ to $94 \pm 2 \mathrm{bpm}$, $(p<0.001)$. LVEDV remained unchanged $(p=0.81)$ and LVESV decreased with $-9 \pm 18 \%(p<0.05)$ causing LVSV to increase with $8 \pm 3 \%(p<0.05)$. RVEDV and RVESV decreased by $-7 \pm 10 \%$ and $-24 \pm 14 \%$ respectively, $(p<0.001)$ and RVSV increased $5 \pm 17 \%$ during exercise although not statistically significant $(p=0.18$ ). Longitudinal contribution to RVSV decreased during exercise by $-6 \pm 15 \%$ $(p<0.05)$ but was unchanged for LVSV $(p=0.74)$. THV decreased during exercise by $-4 \pm 1 \%$, $(p<0.01)$ and total heart volume variation (THW) increased during exercise from $5.9 \pm 0.5 \%$ to $9.7 \pm 0.6 \%(p<0.001)$.

Conclusions: Cardiac volumes and function are significantly altered during supine physical exercise. THV becomes significantly smaller due to decreases in RVEDV whilst LVEDV remains unchanged. THW and consequently radial pumping increases during exercise which may improve diastolic suction during the rapid filling phase.

Keywords: Physiology, Total heart volume variation, Ventricle, Cardiac pumping, Cardiovascular magnetic resonance

\section{Background}

Total heart volume at rest has a strong correlation to peak exercise capacity in healthy normal subjects and athletes $[1,2]$. When going from rest to exercise the normal heart in a sedentary individual can increase its cardiac output from $5 \mathrm{~L} / \mathrm{min}$ to $20-25 \mathrm{~L} / \mathrm{min}$ [3]. This change has been attributed to an increase in heart rate and stroke

\footnotetext{
* Correspondence: hakan.arheden@med.lu.se

${ }^{3}$ Department of Clinical Physiology, Lund University, Lund University Hospital Lund, Lund, Sweden

Full list of author information is available at the end of the article
}

volume. In turn, the stroke volume can increase either by an increase in end-diastolic volume (EDV), decrease in end-systolic volume (ESV), or both. The effects on left ventricular (LV) volumes during physical exercise remain controversial. Previous studies using radionuclide angiography or echocardiography have shown both unchanged and increased LV end-diastolic volumes (LVEDV) during upright and supine exercise compared to resting values in the same position [4-12]. Although most studies show a decrease in ESV during exercise, Sundstedt et al. [12] showed unchanged ESV during supine exercise using echocardiography. Few studies have investigated the

\section{Biomed Central}


effects of exercise on the right ventricle [13,14] and further studies are needed to understand how physical exercise affects left and right cardiac volumes and subsequently the stroke volume (SV).

Ventricular stroke volume is ejected by a combination of longitudinal and radial contraction of the ventricle [15-18]. At rest the longitudinal contribution to SV has been shown to be $60 \%$ for the LV and $80 \%$ for the RV and radial contribution is $40 \%$ and $20 \%$ respectively $[15,17,19]$. It has been shown that during exercise there is a significant increase in the mitral valve displacement during exercise [20]. Longitudinal pumping is calculated as the atrio-ventricular plane displacement (AVPD) multiplied by the short-axis area of the ventricle and an increase in AVPD may therefore affect the longitudinal contribution to SV. Several studies have suggested that at higher heart rates a larger longitudinal contribution may keep the outer volume variation of the heart to a minimum rendering less energy to be wasted on moving surrounding tissues [21-23]. However, this remains to be explored.

Therefore, the aim of this study was to determine left and right ventricular volumes, left and right atrial volumes and total heart volumes as well as longitudinal and radial pumping during rest and physical exercise using cardiovascular magnetic resonance (CMR).

\section{Methods}

This study was approved by the Regional Ethical Review Board in Lund, Sweden and follows the Declaration of Helsinki. All participants provided written informed consent. All CMR examinations were performed at Skane University Hospital Lund, Sweden.

\section{Study population and experimental setup}

Twenty-six healthy volunteers (six women) aged $30 \pm 8$ years (mean \pm SD) (range 19-59) underwent CMR at rest and during exercise with one-legged knee extensions. A custom built MR-compatible ergometer provided concentric resistance during knee extension by a rope and pulley system which was integrated with a mechanically braked flywheel. A strap connected to a variable weight system provided resistance and weight was added to achieve an exercise level at approximately 40 beats per minute (bpm) higher than the subjects' resting heart rate. The subjects were connected at the ankle to the axle of the flywheel by a rope and the extension phase of the exercise turned the flywheel. Gravity returned the leg to the starting position and a gearing system on the axle returned the rope to the starting position at the end of each duty cycle.

\section{Reproducibility of exercise measurements}

Six subjects underwent a total of five CMR scans to investigate the reproducibility of volumetric measurements during exercise and the potential effects of different respiratory phases as well as differences in exercising muscle mass. The scans were divided into two sessions with a 1.5 hour rest outside the scanner between them. Session 1 included a) CMR at rest; b) CMR with 1-legged exercise at end-expiratory breath hold; and c) CMR with 2-legged exercise at end-expiratory breath hold. Session 2 included a) CMR with 1-legged exercise at end-expiratory breath hold; and b) CMR with 1-legged exercise at endinspiratory breath hold with the instructions to keep an open glottis and avoid Valsalva-like increases in intrathoracic pressures.

\section{Cardiac magnetic resonance imaging}

A $1.5 \mathrm{~T}$ scanner (Philips Achieva, Philips, Best, The Netherlands) with a 5 channel cardiac coil was used to scan all subjects in the supine position. A balanced steady-state free-precession (bSSFP) sequence with retrospective ECG gating was used to acquire images of the heart (repetition time typically $3.0 \mathrm{~ms}$, turbo factor 16 , echo time $1.5 \mathrm{~ms}$, flip angle $60^{\circ}$, reconstructed to a spatial resolution of $1.4 \times 1.4 \mathrm{~mm}$, acquired temporal resolution typically $50 \mathrm{~ms}$ reconstructed to $30 \mathrm{~ms}$, and slice thickness $8 \mathrm{~mm}$ with no slice gap). After defining the long-axis orientation of the heart, short-axis images covering the heart from the base of the atria to the apex of the ventricles were obtained. Breath-hold during imaging during exercise was typically $6 \mathrm{~s}$ for long-axis images and 8-10 s for each short-axis slice. An ECGtriggered phase-contrast sequence was used to measure blood flow in the aorta (repetition time $8.6 \mathrm{~ms}$, echo time $6.4 \mathrm{~ms}, 150 \mathrm{~cm} / \mathrm{s}$ velocity encoding, slice thickness $8 \mathrm{~mm}$ ). The measurement plane was positioned perpendicular to the vessel. Heart rate was obtained from the ECG during image acquisition.

\section{Atrial and ventricular volumes}

All measurements were done using the software Segment 1.8 (http://segment.heiberg.se) [24]. Left and right atrial volumes were measured in short-axis images at the time of ventricular end-diastole and ventricular end-systole. Left ventricular mass (LVM), end-diastolic volume (LVEDV), end-systolic volume (LVESV) and stroke volume (LVSV) were measured in short-axis images using planimetry, by manual delineation of endocardial and epicardial borders of the left ventricle. Papillary muscles were not included in LVM measurements. Right ventricular end-diastolic volume (RVEDV), end-systolic volume (RVESV) and stroke volume (RVSV) were measured in short-axis images by manual delineation of the right ventricular endocardial and epicardial border.

Total heart volume (THV) was measured in short-axis images by planimetry [22] and was defined as the volume of all structures within the pericardium, including 
myocardium, blood pool, atria, pericardial fluid and the proximal parts of the great vessels.

\section{Ventricular pumping}

Atrio-ventricular plane displacement (AVPD) was determined from CMR long-axis images as previously described [15]. Longitudinal pumping of the left and right ventricle was calculated as the distance travelled by the AV-plane multiplied by epicardial short-axis area of the ventricle [25]. Radial pumping was determined from the total heart volume variation (THVV) [15]. Longitudinal and radial contribution to SV (\%) was calculated as longitudinal pumping divided by SV and radial pumping divided by SV.

\section{Statistical analysis}

Statistical analysis was performed using SPSS statistics 20 (IBM, Chicago, IL, USA) and a p-value $<0.05$ was considered statistically significant. Paired $t$-tests were used to test for changes between rest and exercise. Wilcoxon non-parametric test was used to test for differences between rest and exercise in the subgroup of six subjects who underwent a total of five scans to investigate the reproducibility of measurements. Results are presented as mean \pm SEM unless stated otherwise. Inter-observer variability was determined for the left ventricular measurements in ten subjects during rest and exercise.

\section{Results}

Subject characteristics are presented in Table 1. All subjects reported to be healthy and none of the subjects showed any signs of cardiac disease on the CMR scan.

Table 1 Subject characteristics and cardiac volumes at rest for men and women (mean \pm SD)

\begin{tabular}{lcc}
\hline & Men $\mathbf{n}=\mathbf{2 0}$ & Women $\mathbf{n}=\mathbf{6}$ \\
\hline Age (years) & $30 \pm 9$ & $29 \pm 8$ \\
Weight $(\mathbf{k g})$ & $78 \pm 12$ & $61 \pm 14$ \\
Height $(\mathbf{m})$ & $1.80 \pm 0.07$ & $1.68 \pm 0.07$ \\
THV $(\mathbf{m L})$ & $861 \pm 145$ & $586 \pm 123$ \\
LVEDV $(\mathbf{m L})$ & $197 \pm 34$ & $148 \pm 36$ \\
RVEDV $(\mathbf{m L})$ & $219 \pm 37$ & $149 \pm 39$ \\
LVSV $(\mathbf{m L})$ & $109 \pm 19$ & $87 \pm 22$ \\
RVSV $(\mathbf{m L})$ & $110 \pm 17$ & $84 \pm 23$ \\
LVM $(\mathbf{g})$ & $126 \pm 22$ & $79 \pm 17$ \\
LAes $(\mathbf{m L})$ & $89 \pm 19$ & $65 \pm 24$ \\
RAes $(\mathbf{m L})$ & $134 \pm 33$ & $90 \pm 22$
\end{tabular}

$\mathrm{g}=$ gram, $\mathrm{kg}=$ kilogram, LAes = left atrial volume measured at ventricular end-systole, LVEDV = left ventricular end-diastolic volume, LVM = left ventricular mass, LVSV $=$ left ventricular stroke volume, $\mathrm{m}=$ metre, $\mathrm{mL}=$ millilitre, RAes = right atrial volume measured at ventricular end-systole, RVEDV = right ventricular end-diastolic volume, RVSV = right ventricular stroke volume, $\mathrm{THV}=$ total heart volume $\mathrm{THVV}=$ total heart volume variation .
In three subjects the same short-axis slice was imaged twice due to difficulties in breath holding during exercise. These extra slices were identified and removed before the images were analysed. Figure 1 and Additional files 1, 2, 3 and 4 show typical examples of the image quality during exercise.

\section{Heart rate and cardiac volumes}

Heart rate increased significantly during exercise from $60 \pm 2$ to $94 \pm 2 \mathrm{bpm}(\mathrm{p}<0.001)$. Left atrial volumes at enddiastole decreased from $39 \pm 3$ to $35 \pm 3 \mathrm{~mL}(\mathrm{p}<0.05)$ as did right atrial end-diastolic volumes from $65 \pm 5$ to $56 \pm 4 \mathrm{~mL}$ $(\mathrm{p}<0.05)$. At ventricular end-systole, where the atria reach their largest volumes, left atrial volumes were unchanged $(84 \pm 4$ and $85 \pm 5 \mathrm{~mL}, \mathrm{p}=0.72)$ and right atrial volume decreased significantly from $124 \pm 7$ to $103 \pm 7 \mathrm{~mL}(\mathrm{p}<0.001)$ with exercise.

Left ventricular EDV remained unchanged during exercise $(186 \pm 8$ to $185 \pm 8 \mathrm{~mL}, \mathrm{p}=0.81)$ and LVESV decreased from $82 \pm 4$ to $74 \pm 4 \mathrm{~mL}(\mathrm{p}<0.05)$ (Figure $2 \mathrm{~A}-\mathrm{B})$. Left ventricular SV increased from $104 \pm 4$ to $111 \pm 5 \mathrm{~mL}$ $(\mathrm{p}<0.05$, Figure $2 \mathrm{C})$. For the right ventricle, both RVEDV and RVESV decreased from $203 \pm 9$ to $185 \pm 10 \mathrm{~mL}$ for RVEDV and from $100 \pm 6$ to $77 \pm 6 \mathrm{~mL}$ for RVESV $(\mathrm{p}<0.001$ for both) (Figure 2D-E) but the increase in right ventricular SV from $104 \pm 4$ to $108 \pm 5 \mathrm{~mL}$ was not significant $(\mathrm{p}=0.18)$ (Figure $2 \mathrm{~F})$. Both left and right ventricular ejection fraction (LVEF and RVEF) increased during exercise from $56 \pm 1$ to $60 \pm 1 \%$ and from $52 \pm 1$ to $59 \pm 1 \%$ respectively ( $<<0.01$ for both). Cardiac output increased from $6.2 \pm 0.3$ to $10.4 \pm 0.5 \mathrm{~L} / \mathrm{min}(\mathrm{p}<0.001)$ mainly due to the increase in heart rate (Figure 3A-B). Interestingly, total heart volume decreased significantly during exercise by $-30 \pm 8 \mathrm{~mL},(\mathrm{p}<0.01)$ corresponding to a $4 \pm 1 \%$ decrease of volume (example shown in Figure 4 and in Additional file 4). As expected, LVM was unchanged from rest to exercise ( $115 \pm 6$ to $114 \pm 6 \mathrm{~g}, \mathrm{p}=0.62)$.

\section{Left and right atrio-ventricular plane displacement}

Left ventricular AVPD and RVAVPD remained unchanged during exercise. Left ventricular AVPD was $14.6 \pm 0.3 \mathrm{~mm}$ at rest and $15.3 \pm 0.5 \mathrm{~mm}$ during exercise $(\mathrm{p}=0.06)$ and the RV AVPD was $20.9 \pm 0.6 \mathrm{~mm}$ for both rest and exercise $(\mathrm{p}=0.90)$.

\section{Longitudinal and radial pumping}

Left ventricular longitudinal contribution to SV (\%) remained unchanged at approximately $60 \%(59 \pm 1 \%$ at rest and $60 \pm 2 \%$ at exercise, $\mathrm{p}=0.74$ ). Right ventricular longitudinal contribution (\%) decreased from $81 \pm 2$ to $75 \pm 2 \%(\mathrm{p}<0.05)$ (Figure 5A-B) due to the decrease in $\mathrm{RV}$ end-diastolic volume. Total heart volume variation increased during exercise from $5.9 \pm 0.5$ to $9.7 \pm 0.6 \%$ $(\mathrm{p}<0.001)$ (Figure 5C). 


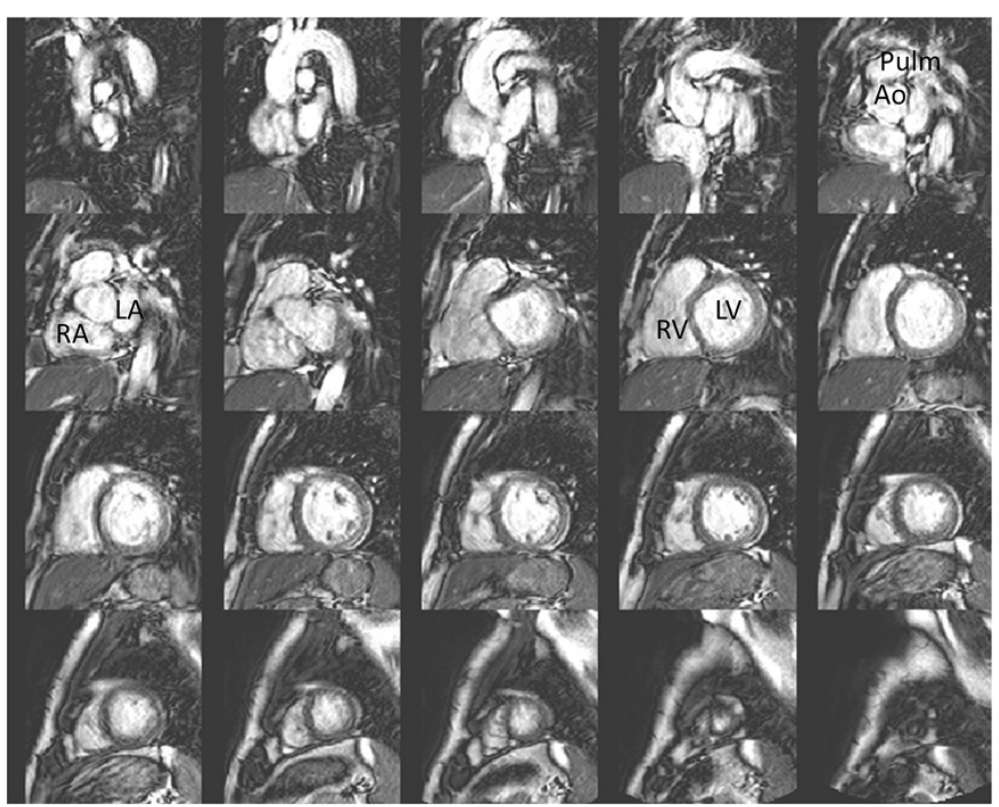

Figure 1 Short-axis images showing the typical image quality during exercise. These images were acquired at a heart rate of $119 \mathrm{bpm}$. The top left image shows the most basal short-axis slice showing the roof of the atria and the bottom right image shows the most apical slice of the ventricles. Ao - aorta, LA -left atrium, LV - left ventricle, Pulm - pulmonary trunk, RA - right atrium, RV - right ventricle.

\section{Reproducibility of exercise measurements}

For the six subjects participating in repeated scans there were no differences in THV, RVEDV or left and right SV between the first and second exercise session with one leg. Left ventricular EDV increased more during the second exercise session when compared to rest; a $5 \pm 5 \%$ increase during the first session and a $11 \pm 4 \%$ increase during the second session $(\mathrm{p}<0.05)$. When comparing endexpiratory breath hold with end-inspiratory breath hold, only LVEDV differed between sessions. When compared
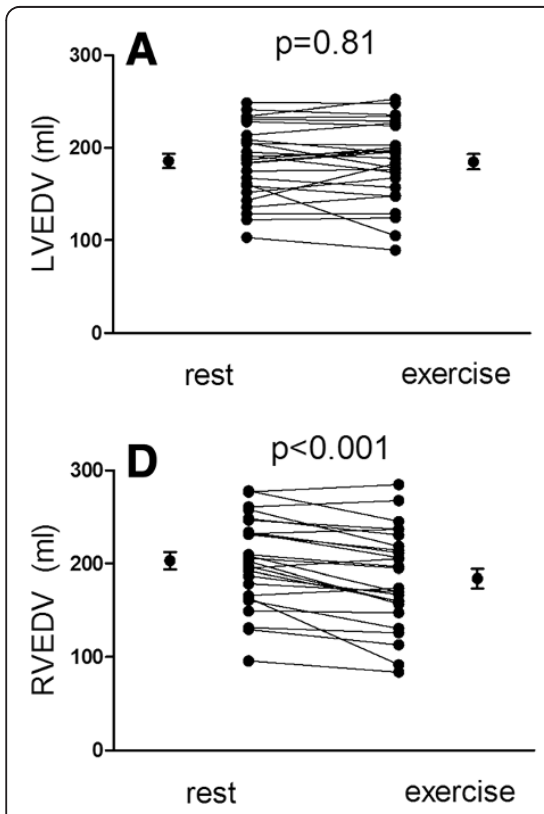
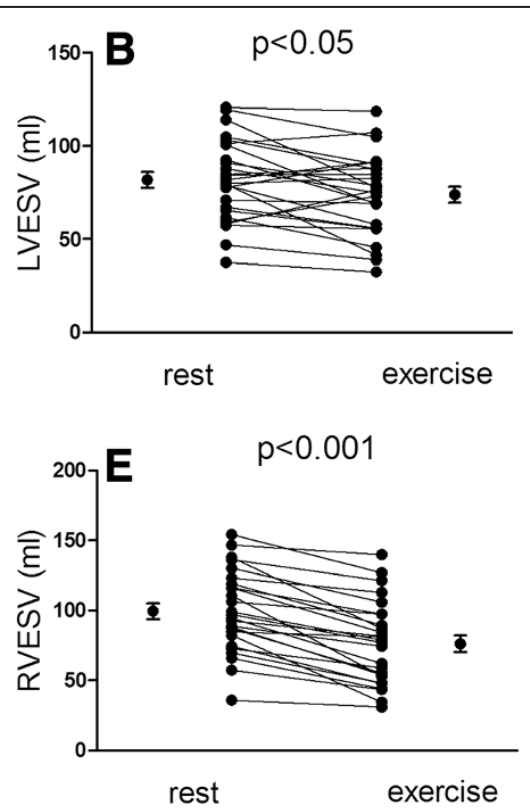
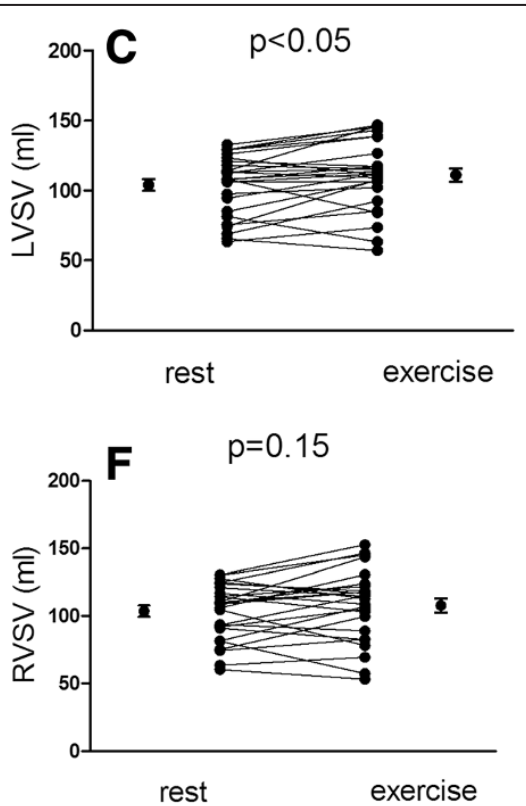

Figure 2 Left and right ventricular volumes and stroke volumes at rest and exercise. Upper panel shows no changes in left ventricular end-diastolic volumes (A) and a small but significant decrease in end-systolic volume (B), leading to an increased stroke volume (C). Lower panel show a significant decrease in right ventricular end-diastolic volume (D) and end-systolic volume (E). Right ventricular stroke volume increased during exercise, however not statistically significant (F). Error bars denote mean and standard error of the mean (SEM). 
A

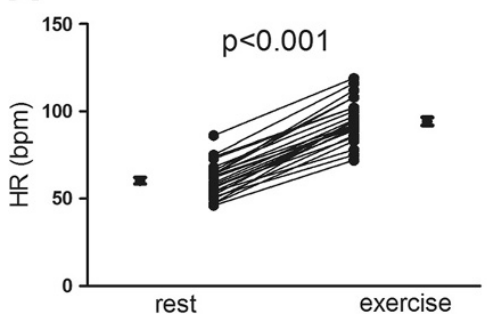

B

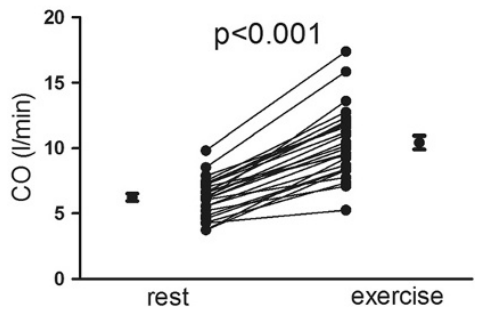

Figure 3 Heart rate and cardiac output at rest and exercise. Heart rate (A) and cardiac output (B) increased significantly from rest to exercise. Error bars denote mean and standard error of the mean (SEM).

to rest there was a $5 \pm 5 \%$ increase at end-expiratory breath hold and a $15 \pm 7 \%$ increase at end-inspiratory breath hold $(\mathrm{p}<0.05)$. During exercise using two legs, left and right ventricular EDV did not differ when compared to one-legged exercise. Left ventricular EDV increased by $5 \pm 5 \%$ and $7 \pm 7 \%$ respectively $(\mathrm{p}=0.35)$, and RVEDV decreased by $-4 \pm 6 \%$ and $-2 \pm 7 \%$ respectively $(\mathrm{p}=0.17)$. Right ventricular $\mathrm{SV}$, however, increased more during exercise using two legs. When compared to rest the increase was $3 \pm 10 \%$ with one leg and $12 \pm 9 \%$ with two legs.

\section{Inter-observer variability and validation}

Results are presented as mean \pm SD. At rest, interobserver variability for LVM was $9 \pm 5$ g, EDV $-1 \pm 4 \mathrm{~mL}$ and $\mathrm{SV}-2 \pm 5 \mathrm{~mL}$. During exercise imaging was more difficult and the image quality was lower, which is reflected by a slightly larger variability; LVM $7 \pm 10 \mathrm{~g}$, EDV $-3 \pm 16 \mathrm{~mL}$ and SV $0 \pm 9 \mathrm{~mL}$.

\section{Discussion}

The present study has shown that the total heart volume decreases in healthy normal subjects during moderate exercise in the supine position. This decrease is caused by reduced right atrial and ventricular volumes whilst left atrial and ventricular volumes remain unchanged during exercise. With regards to pumping function there is an increase in outer volume changes during exercise and thus, an increased radial contribution to stroke volume. AV-plane movement is unchanged during exercise but a smaller short-axis area of the right ventricle causes the lower longitudinal contribution to RVSV. Left ventricular longitudinal contribution to $\mathrm{SV}$ is unchanged during exercise. Total left and right ventricular SV were only slightly increased (LVSV) or unchanged (RVSV) during supine exercise and the increase in cardiac output is best explained by the rise in heart rate.

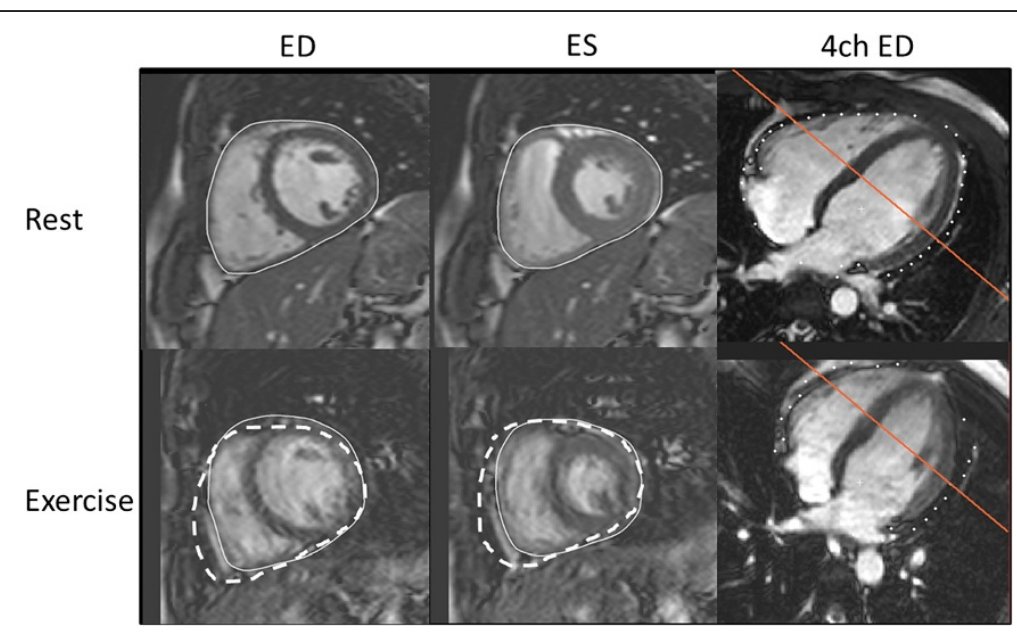

Figure 4 Mid-ventricular short axis slices in end-diastole (ED) and end-systole (ES) during rest and exercise with the corresponding 4-chamber (4 ch) view to illustrate the location of the slice. The solid line indicates delineations for total heart volume. In the exercise images, the dashed line shows the total heart volume delineation copied from the corresponding resting image. The right ventricular volume is decreased whereas the left ventricle remains unchanged. 

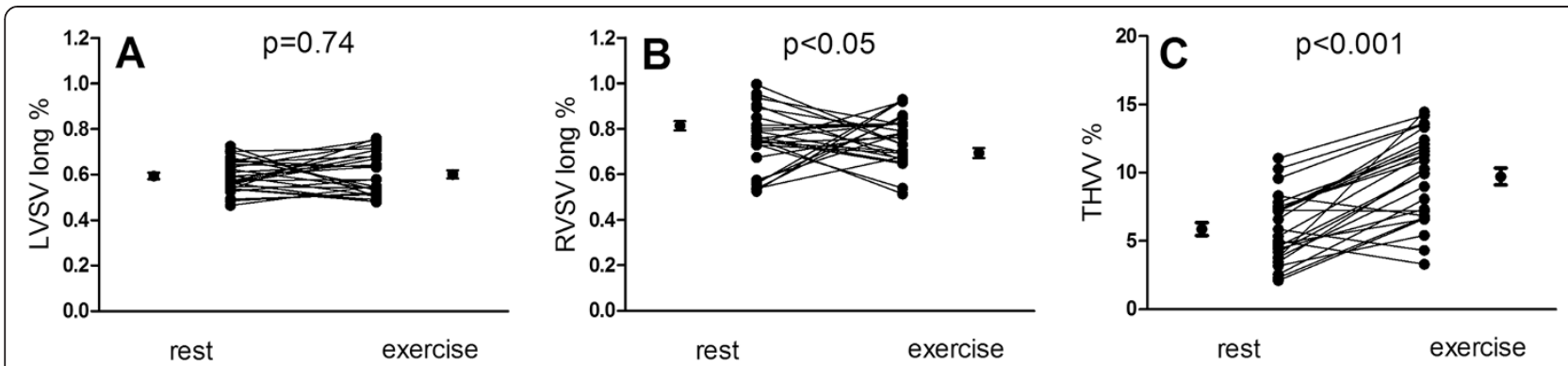

Figure 5 Longitudinal and radial contribution to stroke volume at rest and exercise. Left ventricular longitudinal contribution increased (A) whereas the right ventricular contribution decreased (B). Total radial contribution calculated as total heart volume variation THW (C) increased significantly indicating an overall increase in radial pumping of the heart during exercise. Error bars denotes mean and standard error of the mean (SEM).

\section{Ventricular volumes and stroke volume}

The inconsistent results of previous studies [4-7,11,12,14,26] may be explained by differences in imaging modalities and, perhaps most important, body position. The results of this study are in line with other studies of supine exercise showing unchanged LVEDV $[5,9,10,27,28]$. The significant decrease in RVEDV differs from previous studies of RV volumes during supine exercise using radionuclide ventriculography [13] and CMR [14,28] where RVEDV remained unchanged during moderate intensity exercise (mean HR in these studies were 112,120 and $100 \mathrm{bpm}$ respectively). However, in line with our results the study by Mols et al. [13] used radionuclide ventriculography and showed a decreased RVEDV at workloads at a HR of $127 \mathrm{bpm}$ and above. The differences between the present study and previous CMR studies may be explained by differences in exercise protocol where we acquired breathhold images during leg exercise whilst Holverda et al. [28] used non-breath hold imaging and Roest et al. [14] allowed the subjects to rest for the short period of image acquisition. Free-breathing may decrease image quality and rest during image acquisition will allow HR to decrease making interpretation of results more difficult.

In line with studies by Bevegård et al. [29] the present study showed that $\mathrm{CO}$ increased significantly due to increased HR whilst SV only increased by $8 \%$. During the early stages of exercise in healthy subjects, the increase in HR is primarily caused by a decreased parasympathetic tone whereas the sympathetic effects are not seen until later stages [30]. As the exercise bouts of the study were short, the increased HR with only a small increase in SV is likely caused by parasympathetic withdrawal. Furthermore, the increased venous return caused by the supine position lead to maximal filling of the ventricles already at rest, which would explain the discrepancy between our study and exercise studies performed in the upright position. Our study would then be more representative of exercise in the supine position such as swimming or perhaps in micro gravitational environments such as space flight.

\section{Longitudinal and radial contribution to stroke volume}

In contrast to a previous study of upright exercise on an ergometer cycle where the left ventricular valve displacement was significantly increased during exercise [20], our results showed unchanged LV AVPD and longitudinal contribution to LVSV. Right ventricular valve displacement (RV AVPD) remained unchanged but together with the decreased volume of the right ventricle, the right ventricular longitudinal contribution to SV was significantly decreased. Furthermore, total cardiac pumping became significantly more radial during exercise as shown by the increased THVV when exercising both with one and two legs, as well as during end-expiratory and end-inspiratory breath hold. This is in contrast to a hypothesis previously suggested by our group [22] where we expected cardiac longitudinal pumping to increase and radial pumping to decrease. Increased radial pumping as seen in the present study may theoretically increase the amount of energy spent on moving surrounding tissues and thus decrease the energy efficiency of the heart. However, for the left ventricle, Riordan and Kovács [31] showed that radial pumping may actually be important for diastolic suction during the rapid filling phase. Exercise requires rapid mass transfer from the atria to the ventricle, and it is possible that the increased radial pumping seen in the right ventricle may actually improve cardiac pumping efficiency due to enhanced diastolic suction.

It is possible that our findings of increased THVV only relates to exercise in the supine position such as swimming, and it would be of interest to perform similar studies during upright exercise.

\section{Reproducibility of exercise measurements}

Ventricular volumes and THV were reproducible between the first and second exercise session, and also when imaging was performed at end-inspiratory breath hold as well as during exercise with two legs. The differences seen in LVEDV between the first and second exercise session with one leg as well as between end-expiratory and end- 
inspiratory breath hold is probably best explained by individual variations that are more distinguishable in the small population. As shown in Figure 2 there is some variability between individuals for all variables and when only assessing six subjects results may fall out as statistically significant although not physiologically relevant.

\section{Clinical implication}

Heart failure is a complex syndrome and diagnosis can be especially challenging at early stages. Cardiac MR during physical exercise may become useful for assessing patients with normal ejection fraction and suspected heart failure to investigate if cardiac function and filling are affected during low and medium intensity exercise. Furthermore, exercise CMR may also be used to asses patients with congenital heart disease such as Tetralogy of Fallot before and after surgery.

\section{Limitations}

Exercise heart rate in our healthy volunteers only increased by approximately $40 \mathrm{bpm}$ over resting $\mathrm{HR}$ and it is possible that a higher exercise HR may yield different results. The study population included to test for reproducibility of exercise measurements was small $(n=6)$ and the results of the statistical tests of this subpopulation on reproducibility should be interpreted with caution. Furthermore, the study was performed in the supine position limiting the interpretation of our results to supine exercise such as swimming, but it may also be applicable for conditions of microgravity, such as space flight.

\section{Conclusions}

Moderate intensity exercise in the supine position significantly decreases the total heart volume. This is due to decreases in right atrial and ventricular volumes at end-diastole whilst the LVEDV remains unchanged. The contribution of longitudinal pumping to stroke volume is unchanged in the left ventricle but decreased in the right ventricle in exchange for an increase in radial pumping. In contrast to previous belief, THVV and consequently radial pumping increases which may improve diastolic suction of the ventricles.

\section{Additional files}

Additional file 1: Short-axis image of a healthy heart during exercise at a heart rate of $108 \mathrm{bpm}$.

Additional file 2: Two-chamber long-axis view of a healthy heart during exercise at a heart rate of $102 \mathrm{bpm}$.

Additional file 3: Three-chamber long-axis view of a healthy heart during exercise at a heart rate of $117 \mathrm{bpm}$.

Additional file 4: Four-chamber long-axis view of a healthy heart during exercise at a heart rate of $124 \mathrm{bpm}$.

\section{Competing interests}

The authors declared that they have no competing interest.

\section{Authors' contributions}

KSE: Conception of study, data inclusion and analysis, interpretation of data, drafting and revising the manuscript. RJ: Data inclusion and critical revision of the manuscript. PMA: Data inclusion and analysis, critical revision of the manuscript. MC: Conception of study, data inclusion and critical revision of the manuscript. BS: Conception of study, construction of MR ergometer, critical revision of manuscript. HA: Conception of study, critical revision of manuscript. All authors read and approved the final manuscript.

\section{Acknowledgement}

The authors wish to thank FlemmingJessen at the Copenhagen Muscle Research Centre for design and construction of the ergometer and Ance Kreslin for help with data collection and analysis. This study was supported by the Swedish Research Council, the Swedish Heart and Lung Foundation, Region of Scania, the Medical Faculty at Lund University, Sweden, the Swedish Heart Association and Novo Nordisk Foundation, Denmark.

\section{Author details}

${ }^{1}$ Copenhagen Muscle Research Centre, Copenhagen, Denmark. ${ }^{2}$ Danish Research Centre for Magnetic Resonance, Hvidovre Hospital, Copenhagen, Denmark. ${ }^{3}$ Department of Clinical Physiology, Lund University, Lund University Hospital Lund, Lund, Sweden.

Received: 26 April 2013 Accepted: 1 October 2013

Published: 24 October 2013

\section{References}

1. Steding K, Engblom H, Buhre T, Carlsson M, Mosen H, Wohlfart B, Arheden H. Relation between cardiac dimensions and peak oxygen uptake. J Cardiovasc Magn Reson. 2010; 12(1):8.

2. Henschen ES. Skiddlauf und skidwettlauf. Eine medizinische sportstudie. Mitt. Med. Klin. Upsala: Jena Fischer Verlag; 1899.

3. Widmaier E, Raff H, Strang K. Human Physiology. The Mechanisms of Body Function. 11 th ed. New York: McGRaw-Hill; 2008.

4. Bar-Shlomo BZ, Druck MN, Morch JE, Jablonsky G, Hilton JD, Feiglin DH, McLaughlin PR. Left ventricular function in trained and untrained healthy subjects. Circulation. 1982; 65(3):484-8.

5. Crawford MH, White DH, Amon KW. Echocardiographic evaluation of left ventricular size and performance during handgrip and supine and upright bicycle exercise. Circulation. 1979; 59(6):1188-96.

6. Fagard R, Van den Broeke C, Amery A. Left ventricular dynamics during exercise in elite marathon runners. J Am Coll Cardiol. 1989; 14(1):112-8.

7. Henriksen E, Sundstedt M, Hedberg P. Left ventricular end-diastolic geometrical adjustments during exercise in endurance athletes. Clin Physiol Funct Imaging. 2008; 28(2):76-80.

8. Rerych SK, Scholz PM, Sabiston DC Jr, Jones RH. Effects of exercise training on left ventricular function in normal subjects: a longitudinal study by radionuclide angiography. Am J Cardiol. 1980; 45(2):244-52.

9. Stein RA, Michielli D, Diamond J, Horwitz B, Krasnow N. The cardiac response to exercise training: echocardiographic analysis at rest and during exercise. Am J Cardiol. 1980; 46(2):219-25.

10. Stein RA, Michielli D, Fox EL, Krasnow N. Continuous ventricular dimensions in man during supine exercise and recovery. An echocardiographic study. Am J Cardiol. 1978; 41(4):655-60.

11. Sundstedt $M$, Hedberg $P$, Jonason T, Ringqvist I, Brodin LA, Henriksen E. Left ventricular volumes during exercise in endurance athletes assessed by contrast echocardiography. Acta Physio/ Scand. 2004; 182(1):45-51.

12. Sundstedt M, Jonason T, Ahren T, Damm S, Wesslen L, Henriksen E. Left ventricular volume changes during supine exercise in young endurance athletes. Acta Physio/ Scand. 2003; 177(4):467-72.

13. Mols $P$, Huynh $\mathrm{CH}$, Naeije N, Ham HR. Volumetric response of right ventricle during progressive supine exercise in men. Am J Physiol. 1991; 261(3 Pt 2):H751-4.

14. Roest AA, Kunz P, Lamb HJ, Helbing WA, van der Wall EE, De Roos A Biventricular response to supine physical exercise in young adults assessed with ultrafast magnetic resonance imaging. Am J Cardiol. 2001; 87(5):601-5 
15. Carlsson M, Ugander M, Heiberg E, Arheden H. The quantitative relationship between longitudinal and radial function in left, right, and total heart pumping in humans. Am J Physiol Heart Circ Physiol. 2007; 293(1):H636-44

16. Lundbäck S. Cardiac pumping and function of the ventricular septum. Acta Physiol Scand. 1986; 127:8-101.

17. Steding-Ehrenborg K, Carlsson M, Stephensen SS, Arheden H. Atrial aspiration from pulmonary and caval veins is caused by ventricular contraction and secures $70 \%$ of the total stroke volume independent of resting heart rate and heart size. Clin Physiol Funct Imaging. 2013; 33(3):233-40.

18. Waters EA, Bowman AW, Kovacs SJ. MRI-determined left ventricular "crescent effect": a consequence of the slight deviation of contents of the pericardial sack from the constant-volume state. Am J Physiol Heart Circ Physiol. 2005; 288(2):H848-53.

19. Carlhall CJ, Lindstrom L, Wranne B, Nylander E. Atrioventricular plane displacement correlates closely to circulatory dimensions but not to ejection fraction in normal young subjects. Clinical physiology. 2001; 21(5):621-8.

20. Sundstedt $M$, Hedberg $P$, Henriksen E. Mitral annular excursion during exercise in endurance athletes. Clin Physiol Funct Imaging. 2008; 28(1):27-31.

21. Brecher GA. Cardiac variations in venous return studied with a new bristle flowmeter. Am J Physiol. 1954; 176(3):423-30.

22. Carlsson M, Cain P, Holmqvist C, Stahlberg F, Lundback S, Arheden H. Total heart volume variation thoughout the cardiac cycle in humans. Am J Physiol Heart Circ Physiol. 2004; 287:243-50.

23. Gauer $\mathrm{OH}$. Volume changes of the left ventricle during blood pooling and exercise in the intact animal; their effects on left ventricular performance. Physiol Rev. 1955; 35(1):143-55.

24. Heiberg E, Sjogren J, Ugander M, Carlsson M, Engblom H, Arheden $H$. Design and validation of Segment - freely available software for cardiovascular image analysis. BMC Med Imaging. 2010; 10(1):1

25. Carlsson M, Ugander M, Mosen $H$, Buhre T, Arheden H. Atrioventricular plane displacement is the major contributor to left ventricular pumping in healthy adults, athletes, and patients with dilated cardiomyopathy. Am J Physiol Heart Circ Physiol. 2007; 292(3):H1452-9.

26. Widmaier E, Raff H, Strang K. Mechanical events of the cardiac cycle. In: Human Physiology - The Mechanisms for Body Function. 10th ed. New York: McGraw-Hill Higher Education; 1996: p. 404-5.

27. Schairer JR, Stein PD, Keteyian S, Fedel F, Ehrman J, Alam M, Henry JW Shaw $T$. Left ventricular response to submaximal exercise in endurancetrained athletes and sedentary adults. Am J Cardiol. 1992; 70(9):930-3.

28. Holverda S, Gan CT, Marcus JT, Postmus PE, Boonstra A, Vonk-Noordegraaf A. Impaired stroke volume response to exercise in pulmonary arterial hypertension. J Am Coll Cardiol. 2006; 47(8):1732-3.

29. Bevegard S. Studies on the regulation of the circulation in man. With special reference to the stroke volume and the effect of muscular work body position and artificially induced variations of the heart rate. Acta physiologica Scandinavica Supplementum. 1962; 57(200):1-36.

30. Chaitman BR. Should early acceleration of heart rate during exercise be used to risk stratify patients with suspected or established coronary artery disease? Circulation. 2007; 115(4):430-1

31. Riordan MM, Kovacs SJ. Relationship of pulmonary vein flow to left ventricular short-axis epicardial displacement in diastole: model-based prediction with in vivo validation. Am J Physiol Heart Circ Physiol. 2006; 291(3):H1210-5.

doi:10.1186/1532-429X-15-96

Cite this article as: Steding-Ehrenborg et al: Moderate intensity supine exercise causes decreased cardiac volumes and increased outer volume variations: a cardiovascular magnetic resonance study. Journal of Cardiovascular Magnetic Resonance 2013 15:96.

\section{Submit your next manuscript to BioMed Central and take full advantage of:}

- Convenient online submission

- Thorough peer review

- No space constraints or color figure charges

- Immediate publication on acceptance

- Inclusion in PubMed, CAS, Scopus and Google Scholar

- Research which is freely available for redistribution 